\title{
Influência da maceração carbônica e da irradiação ultravioleta nos níveis de trans-resveratrol em vinhos de uva cabernet sauvignon
}

\author{
Silvana Maria Michelin Bertagnolli*, Simone Bertazzo Rossato, Vagner Lopes Silva, Taciana \\ Cervo, Cláudia Kachler Sautter, Luisa Helena Hecktheuer, Neidi Garcia Penna
}

Departamento de Tecnologia e Ciência de alimentos, Universidade Federal de Santa Maria

*Correspondência:

S. M. M. Bertagnolli

Departamento de Tecnologia e

Ciência de Alimentos

Universidade Federal de Santa Maria Av. Roraima, s/no

97105-900 - Santa Maria - RS, Brazil

E-mail: ngpenna@smail.ufsm.br
O consumo moderado de vinho reduz significativamente os riscos de doenças cardiovasculares. Este efeito é atribuido aos polifenóis presentes no vinho, em especial ao resveratrol (3,5,4'triidroxiestilbeno), que é uma fitoalexina encontrada em várias partes da videira, principalmente na casca da uva, assim como em outras espécies de plantas. Uvas da cultivar Cabernet Sauvignon foram submetidas à irradiação com luz ultravioleta e maceração carbônica e após fermentadas. Procedeu-se à coleta de amostras durante todo o experimento, as quais foram posteriormente analisadas quanto ao teor de trans-resveratrol através da Cromatografia Líquida de Alta Eficiência. Os resultados do trabalho demonstram que a evolução do conteúdo de transresveratrol foi ascendente durante as fases da fermentação. Diferenças ocorreram no final da fermentação, em que as amostras de vinhos com maceração carbônica apresentaram leve declínio, possivelmente pela atmosfera de $\mathrm{CO}_{2}$ na qual ficaram armazenadas, inibindo a formação do resveratrol. Ao final da fermentação principal a concentração de trans-resveratrol foi de $15 \mathrm{mg} . \mathrm{L}^{-1}$ em todos os tratamentos, chegando a 1,5 mg. $\mathrm{L}^{-1}$, em média, no final do periodo de estocagem.
Unitermos

- Resveratrol

- Irradiação UV

- Maceração carbônica

- Cabernet sauvignon

\section{INTRODUÇÃO}

Estudos desenvolvidos no mundo inteiro comprovam que o vinho tomado em quantidade moderada contribui para a saúde do organismo humano (Tomera, 1999). Sugere-se que o resveratrol pode ser um dos constituintes do vinho que conferem esta proteção (Adrian et al., 2000). O resveratrol age na prevenção de doenças cardíacas, associada com o consumo de vinho tinto, assim como na inibição da agregação plaquetária, alteração na síntese de eucosanóides e por modular o metabolismo de lipídios e lipoproteínas (Soleas et al., 1997). A presença de transresveratrol na medicina popular japonesa tem levado a um número de experimentos em animais sugerindo que ele tem atividade antiinflamatória e anticoagulante que protegem contra aterosclerose e doenças coronarianas (Arichi et al., 1982; Kimura et al., 1983). Além disso, o consumo de vinho tem sido relacionado à redução da ocorrência de câncer e doenças degenerativas, como Alzheimer e demência (Dorozynski, 1997). 
As análises de resveratrol na casca e polpa de Vitis vinifera em vinhos têm ganhado aumento considerável de pesquisas nos últimos anos (Roggero, GarciaParrilla,1995). O resveratrol (3,5,4'-triidroxiestilbeno) é uma fitoalexina, ou seja, um polifenol de defesa, encontrado em várias partes da videira, principalmente na casca da uva, assim como em outras espécies de plantas (Soleas et al.,1995). Esta fitoalexina é sintetizada na videira em resposta à infecção por fungos ou "stress" (desordem metabólica) abiótico (Jeandet et al.,1995).

No grão de uva, a síntese de resveratrol é principalmente iniciada na casca e é ausente ou em baixíssima concentração na polpa da fruta (Jeandet et al.,1991; Kallithraka et al., 2001). Na vinificação de uvas tintas, a maceração com cascas e sementes durante a fermentação é o principal fator pelos altos níveis de resveratrol nos vinhos tintos, quando comparados a vinhos brancos (Lamuela Raventos et al., 1995; Soleas et al.,1995; Kallithraka et al., 2001). A concentração de resveratrol aumenta durante a fermentação em presença da casca, mas esta quantidade é dependente da variedade de uva e das condições enológicas (Soleas et al.,1995; Okuda, Yokotsuka, 1996; Lamuela-Raventos et al., 1997; Kallithraka et al.,2001). A extração do resveratrol da casca pode ser facilitada com o etanol produzido durante o processo de fermentação (Threlfall et al., 1999).

Uma das condições da viticultura que influenciam o conteúdo de resveratrol é a irradiação ultravioleta (UV) emitida pelo sol. A incidência desta radiação nos tecidos de plantas apresenta efeito importante sobre o metabolismo fenólico. A luz UV do tipo B está associada com o aumento das enzimas responsáveis pela biossíntese de flavonóides, os quais podem proteger a uva da injúria por raios UV, prevenindo o dano ao material genético da planta (Cantos et al., 2000; Cantos et al., 2003). A luz UV do tipo C também produz um "stress" abiótico nos tecidos da planta e afeta o metabolismo fenólico em diferentes vias, tanto na síntese de resveratrol como na síntese de chalcona e seus derivados, sendo eles flavonóides, antocianinas e compostos aromáticos (Sautter, 2003).

Mattivi e Nicolini (1993) estudaram condições de vinificação que influenciam o conteúdo de trans-resveratrol como o sistema de manejo completo, maceração carbônica, hiperoxidação de mostos e condições de estocagem.

A maceração carbônica é uma técnica de fabricação de vinhos usada principalmente, na produção de vinhos tintos, porém, como já foi mencionado, também na produção de vinhos brancos (Silva et al., 1993). Este processo usa a transformação enzimática natural que ocorre rapidamente na casca em condições anaeróbicas sobre $\mathrm{CO}_{2}$ (Jeandet et al., 1995). A baga sofre fermentação intracelular, em que o ácido málico, principal composto metabolizado, é transformado em álcool etílico e em outras substâncias (Rizzon et al., 1999).

Muitos artigos têm sido publicados descrevendo algumas estimativas para avaliar o conteúdo de cis e transresveratrol em vinhos produzidos por diferentes variedades de uvas (Goldberg et al., 1995; Rodríguez-Delgado et al., 2002). Segundo Souto et al (2001) vinhos de diversos países apresentaram valores médios de resveratrol: Canadá, 0,77 mg. $\mathrm{L}^{-1}$, EUA, 0,998 mg.L $\mathrm{L}^{-1}$, Grécia, 0,873 mg.L-1, Japão, 0,157 mg.L $\mathrm{L}^{-1}$, Portugal, 1,00 mg.L $\mathrm{L}^{-1}$, Chile e Argentina, 1,21 mg.L $\mathrm{L}^{-1}$. No Brasil, existem poucos estudos sobre o conteúdo de resveratrol em vinhos. Souto et al. (2001) apresentaram valores para resveratrol em vinhos produzidos na região Sul do País, sendo que em vinhos de uva Cabernet Sauvignon a média dos valores foi de 1,78 mg.L ${ }^{1}$ de trans-resveratrol.

Este trabalho tem como objetivo determinar o conteúdo de trans-resveratrol em vinhos tintos utilizando técnicas de pós-colheita como irradiação UV-C seguida de vinificação e maceração carbônica para verificar se houve aumento no conteúdo de resveratrol em relação à vinificação tradicional.

\section{MATERIAL E MÉTODOS}

A uva Cabernet Sauvignon, cedida pela Vinícola Velho Amâncio de Santa Maria, RS, foi colhida em fevereiro de 2003, na Serra Gaúcha, e transportada em caixas de plástico até a Universidade Federal de Santa Maria (UFSM), ficando armazenadas à temperatura de $0,5^{\circ} \mathrm{C}$, por sete dias, quando foram divididas em duas partes. Uma parte das uvas sofreu irradiação ultravioleta C (UV-C). A outra parte foi novamente dividida pela metade. Destas, uma parte voltou à câmara fria e a outra sofreu maceração carbônica. As uvas que sofreram irradiação também foram divididas em duas partes, sendo que uma voltou à câmara fria e a outra sofreu maceração carbônica. A maceração carbônica foi realizada armazenando-se as uvas em frascos de $10 \mathrm{~kg}$, em atmosfera de $\mathrm{CO}_{2}(10 \%)$, e monitorada com auxílio de analisador de gases e corrigida diariamente. As uvas que ficaram armazenadas em câmara fria permaneceram em caixas de plásticos até o início da vinificação.

O delineamento experimental foi inteiramente casualizado com quatro tratamentos e três repetições, sendo que os tratamentos aplicados foram: controle (C), irradiação (I), maceração carbônica (MC) e irradiação seguida de maceração carbônica (IMC). A unidade amostral foi de $10 \mathrm{~kg}$ de uvas. Os tratamentos aplicados nas uvas, bem como as condições de armazenamento destas, estão apresentados na Tabela I. 
TABELA I - Tratamentos aplicados e condições de armazenamento das uvas

\begin{tabular}{lc}
\hline Tratamento & $\begin{array}{c}\text { Condição de } \\
\text { armazenamento }\end{array}$ \\
\hline Controle (C) & $0,5^{\circ} \mathrm{C}$ \\
Irradiação UV-C 15840 J.m ${ }^{-2}(\mathrm{I})$ & $0,5{ }^{\circ} \mathrm{C}$ \\
Maceração carbônica (MC) & $0,5{ }^{\circ} \mathrm{C} \mathrm{e} \mathrm{CO} 210 \%$ \\
Irradiação UV-C 15840 J.m ${ }^{-2}$ & \\
e maceração carbônica (IMC) & $0,5{ }^{\circ} \mathrm{C} \mathrm{e} \mathrm{CO} 10 \%$ \\
\hline
\end{tabular}

Após o término dos tratamentos, as uvas foram pesadas, separadas da ráquis e maceradas. Em seguida, foi feita a correção do açúcar, a sulfitagem e a adição de levedura comercial para proceder à fermentação. A fermentação foi acompanhada através de leituras periódicas de temperatura e sólidos solúveis totais. Quando a fermentação principal chegou ao final, realizou-se a primeira trasfega. Após o término da fermentação secundária, realizou-se a segunda trasfega. Antes de proceder ao engarrafamento do vinho foi feita mais uma sulfitagem. O vinho após o engarrafamento foi estocado por dois meses.

Foram coletadas amostras das cascas das uvas, do mosto antes de iniciar a fermentação, durante a fermentação principal a cada diminuição de $\pm 3^{\circ}$ Brix, após a primeira trasfega, antes do engarrafamento do vinho e após a estocagem por dois meses.

A extração do resveratrol das cascas foi feita segundo adaptação do método de Cantos et al. (2000), em que as cascas das uvas foram pesadas, adicionado $3 \mathrm{~mL} \cdot \mathrm{g}^{-1} \mathrm{da}$ solução de metanol com $3 \%$ de ácido fórmico e trituradas em Ultra Turrax a $24.000 \mathrm{rpm}$, por um minuto. O extrato foi centrifugado a $5000 \mathrm{G} / 10 \mathrm{~min} /-5^{\circ} \mathrm{C}$ em centrífuga 5403 da marca Eppendorf ${ }^{\circledR}$, sendo que o sobrenadante foi coletado e armazenado a $5{ }^{\circ} \mathrm{C}$.

As demais amostras (mosto e vinho) foram centrifugadas a $5000 \mathrm{G} / 10 \mathrm{~min} /-5^{\circ} \mathrm{C}$ em centrífuga 5403 da marca Eppendorf ${ }^{\circledR}$, sendo que o sobrenadante foi coletado e armazenado a $5{ }^{\circ} \mathrm{C}$, para posterior análise.

Após a finalização de todas as vinificações, as amostras foram novamente centrifugadas da mesma maneira, conforme já descrito, sendo o sobrenadante filtrado a 0,22 mm e mantido a $5{ }^{\circ} \mathrm{C}$. No preparo para análise do resveratrol, a amostra foi homogeneizada em banho de ultra-som Odontobras ${ }^{\circledR}$ por 10 minutos, $500 \mathrm{~mL}$ foram coletados e transferidos para vial recoberto com alumínio e analisado em Cromatografia Líquida de Alta Eficiência (CLAE), em cromatógrafo líquido Agilent ${ }^{\circledR}$ equipado com bomba quaternária de solvente (HP 1100) operando de modo isocrático com injetor automático programado para $10 \mathrm{~mL}$, detector ultravioleta (UV) e detector com arranjo de diodos (DAD) acoplado a um sistema de integração ChemStation ${ }^{\circledR}$. A coluna analítica utilizada foi Merck ${ }^{\circledR}$ C-18 (250 nm x 4,6 $\mathrm{mm}, 5 \mathrm{~mm}$ ) precedida a uma coluna de guarda da mesma composição. A fase móvel foi acetonitrila:água (25:75), $\mathrm{pH}$ 3,0 , corrigido com ácido ortofosfórico, temperatura de $50^{\circ} \mathrm{C}$ numa vazão de $1,5 \mathrm{~mL} / \mathrm{min}$ e detectado a $306 \mathrm{~nm}$. O cromatograma do resveratrol esta apresentado na Figura 1. A identificação foi baseada no tempo de retenção UV e a pureza dos picos foi confirmada através do detector com arranjos de diodos (DAD) (Sautter et al., 2005).

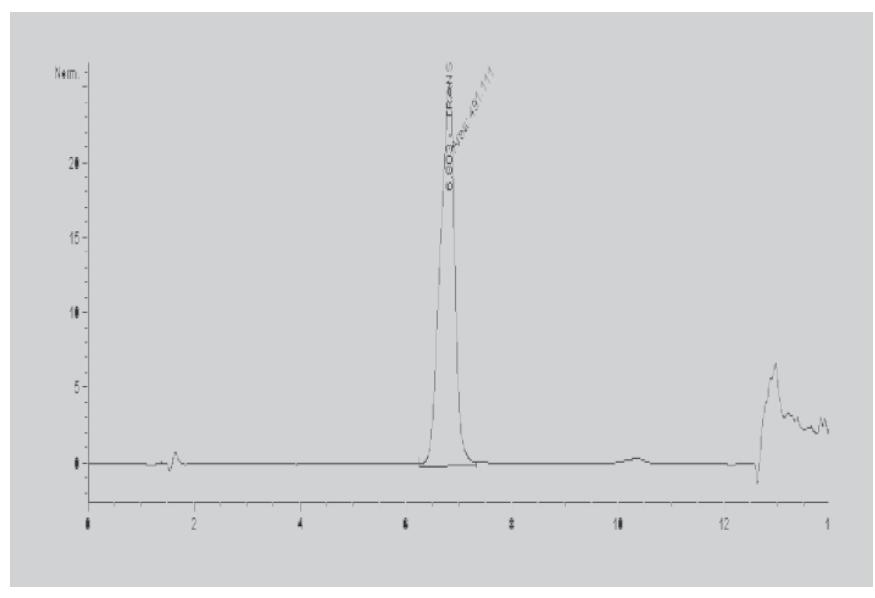

FIGURA 1 - Cromatograma do padrão de trans-resveratrol por CLAE

A quantificação foi baseada na curva de calibração do padrão de trans-resveratrol da marca ICN Biomedicals $100 \mathrm{mg}$, abrangendo as seguintes concentrações: 1,$0 ; 3,0$; 5,$0 ; 8,0 ; 10,0 \mathrm{mg} / \mathrm{L}$, expressando os valores em mg.L.

Os resultados obtidos foram analisados estatisticamente através da análise de variância (ANOVA) e logo em seguida, foi aplicado o teste de Duncan para comparação entre as médias obtidas.

\section{RESULTADOS E DISCUSSÃO}

A evolução de trans-resveratrol durante a fermentação no tratamento controle, ou seja, de uvas não irradiadas e sem maceração carbônica (controle), pode ser observada na Figura 2, por meio da qual pode-se constatar que os valores deste composto tendem a aumentar à medida que $\mathrm{a}$ fermentação ocorre. Comportamento semelhante foi observado no tratamento de uvas irradiadas e sem maceração carbônica (Figura 3).

Nos tratamentos nos quais foi realizada a maceração carbônica, observa-se que a concentração de transresveratrol aumentou nos primeiros dias de fermentação, mas começou a diminuir após três (Figura 4) e seis dias (Figura 5) de fermentação. Este comportamento deve-se, 


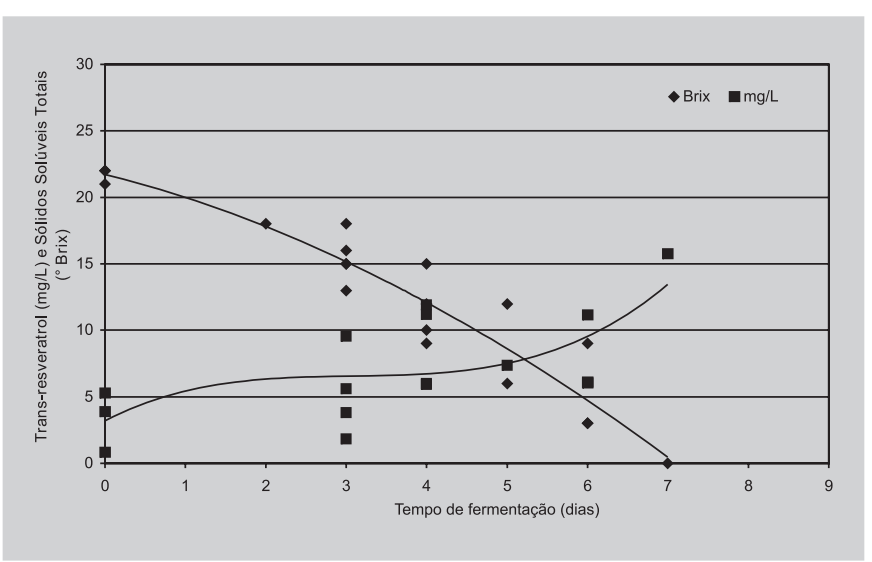

FIGURA 2 - Produção de trans-resveratrol em função do tempo de fermentação em uva Cabernet Sauvignon (Safra 2003) não-irradiada e sem maceração carbônica (controle).

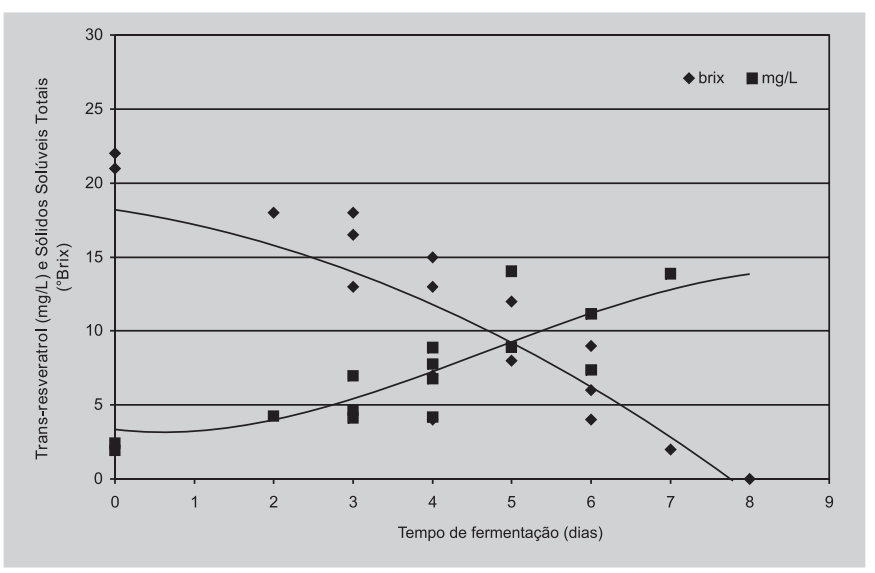

FIGURA 3 - Produção de trans-resveratrol em função do tempo de fermentação em uva Cabernet Sauvignon

provavelmente, à atmosfera de $\mathrm{CO}_{2}$ à qual foram submetidos os mostos. A alta atmosfera de $\mathrm{CO}_{2}$ pode ter inibido a rota de formação do resveratrol, pois para a síntese de cada molécula desta substância deveriam ser eliminadas quatro moléculas de $\mathrm{CO}_{2}$ (Sautter, 2003).

Observa-se no tratamento controle e naquele em que as uvas foram submetidas somente à irradiação, que o ápice das curvas de trans-resveratrol situa-se entre 5 e $10^{\circ}$ Brix, concordando com os dados da literatura, que apresentam uma correlação inversa entre a produção de transresveratrol e sólidos solúveis totais (Lanckage, Price, 1977). Observa-se, também, que as quantidades que aparecem no final da fermentação principal foram, em média, de $15 \mathrm{mg} . \mathrm{L}^{-1}$, maiores do que as encontradas nos mostos correspondentes (2,795 mg.L $\mathrm{L}^{-1} \mathrm{em}$ média), em todos os tratamentos.

No tratamento em que se usaram maceração carbônica e irradiação, observou-se redução do tempo de

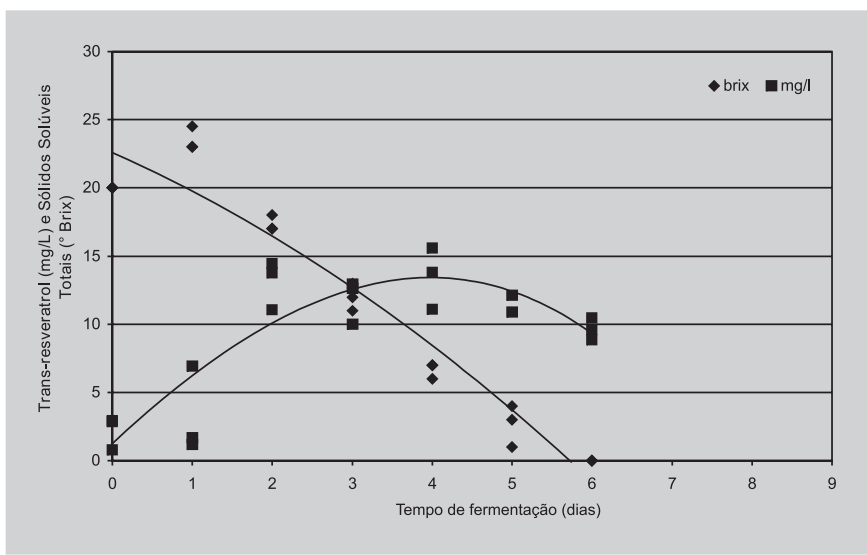

FIGURA 4 - Produção de trans-resveratrol em função do tempo de fermentação em uva Cabernet Sauvignon (Safra 2003) irradiada e com maceração carbônica (IMC).

fermentação (Figura 4), concordando com Rizzon et al. (1999), quando observaram que os vinhos produzidos por maceração carbônica ficaram prontos em menor período de tempo. Isto porque a baga sofre fermentação intracelular, em que o ácido málico, principal composto metabolizado, é transformado em álcool etílico e em outras substâncias.

Os dados referentes à concentração de transresveratrol na casca da uva nos diferentes tratamentos estão apresentados na Figura 6, constatou-se diferença estatística entre todos os tratamentos. As concentrações de trans-resveratrol na casca das uvas não-irradiadas e com maceração carbônica foi a menor $\left(4,25 \mathrm{mg} . \mathrm{L}^{-1}\right)$, quando comparada com os outros tratamentos $(6,49,8,09$ e $14,85 \mathrm{mg} . \mathrm{L}^{-1}$, respectivamente, nos vinhos de uvas irradiadas e com maceração carbônica, vinhos de uvas não-irradiadas e sem maceração carbônica e vinhos de uvas irradiadas e sem maceração carbônica). A maior concentração de trans-resveratrol foi observada nas cascas das uvas irradi-

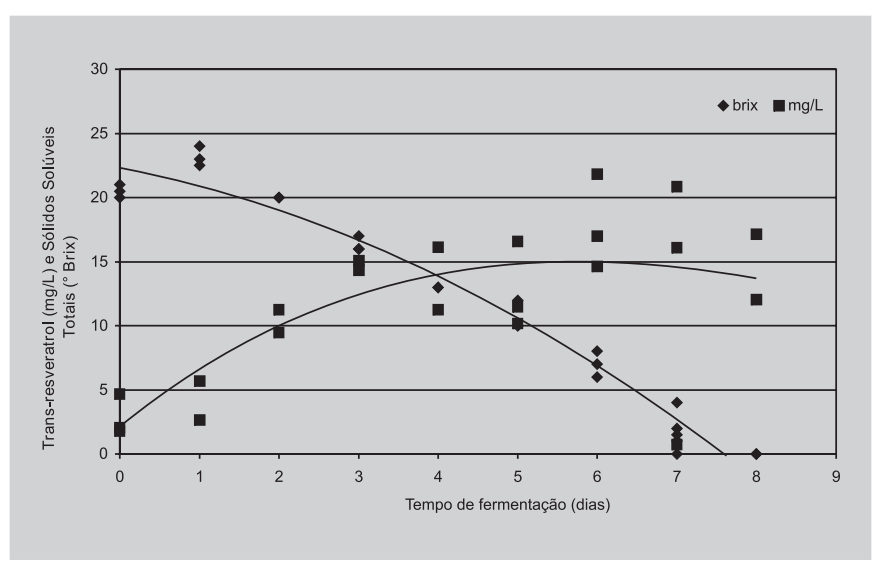

FIGURA 5 - Produção de trans-resveratrol em função do tempo de fermentação em uva Cabernet Sauvignon (Safra 2003) não-irradiadas e com maceração carbônica (MC). 
adas. Porém, quando se associaram a irradiação e a maceração carbônica, a concentração de trans-resveratrol foi menor, quando comparado a uva sem nenhum tipo de tratamento, e maior que nas uvas com maceração carbônica. Comprovou-se então, que a irradiação aumentou a concentração de trans-resveratrol em cascas de uvas pelo "stress" abiótico nos tecidos da planta, que afeta o metabolismo fenólico em diferentes vias (Cantos et al., 2000). Este fato comprova que a irradiação aumenta a concentração de trans-resveratrol nas cascas, coincidindo com dados previamente descritos por Sautter et al. (2005), que determinaram a influência da irradiação na concentração de trans-resveratrol em sucos de uvas. Os baixos valores de resveratrol encontrados quando se utilizou a maceração carbônica reforçam a hipótese de que esta inibiu a formação de resveratrol, uma vez que para sua formação deveriam ser eliminadas moléculas de $\mathrm{CO}_{2}$.

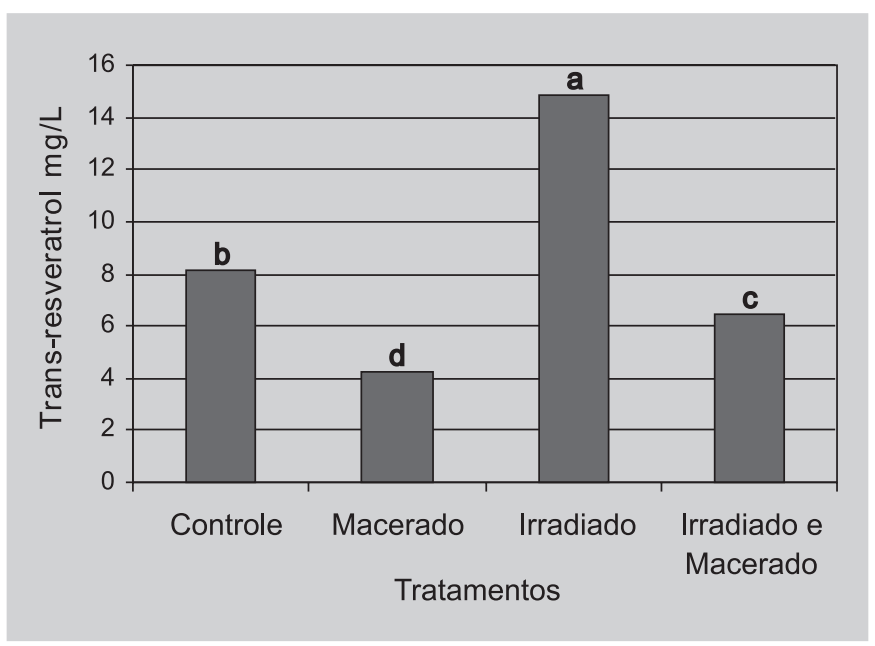

FIGURA 6 - Conteúdo de trans-resveratrol na casca da Cabernet Sauvignon (Safra 2003), submetida a diferentes tratamentos. Letras iguais não diferem significativamente a $5 \%$ de probabilidade pelo teste de Duncan.

A Figura 7 apresenta o conteúdo de trans-resveratrol no vinho após a fermentação principal, no qual procedeuse a operação de trasfega. Nesta etapa da vinificação, as concentrações da substância estão no ápice em todos os tratamentos, inclusive sem diferença estatística entre eles, apresentando valores de 12,19 mg.L $\mathrm{L}^{-1}, 18,11 \mathrm{mg} \cdot \mathrm{L}^{-1}, 16,97$ mg.L $\mathrm{L}^{-1}$ e $12,47 \mathrm{mg} . \mathrm{L}^{-1}$, respectivamente, para os tratamentos com uvas não irradiadas e sem maceração carbônica (controle), somente com maceração carbônica, somente irradiadas e irradiadas e com maceração carbônica. Este fato ocorre devido ao esmagamento do bagaço realizado antes da primeira trasfega para maior rendimento, ocorrendo maior liberação de resveratrol da casca, o qual passa para o vinho.

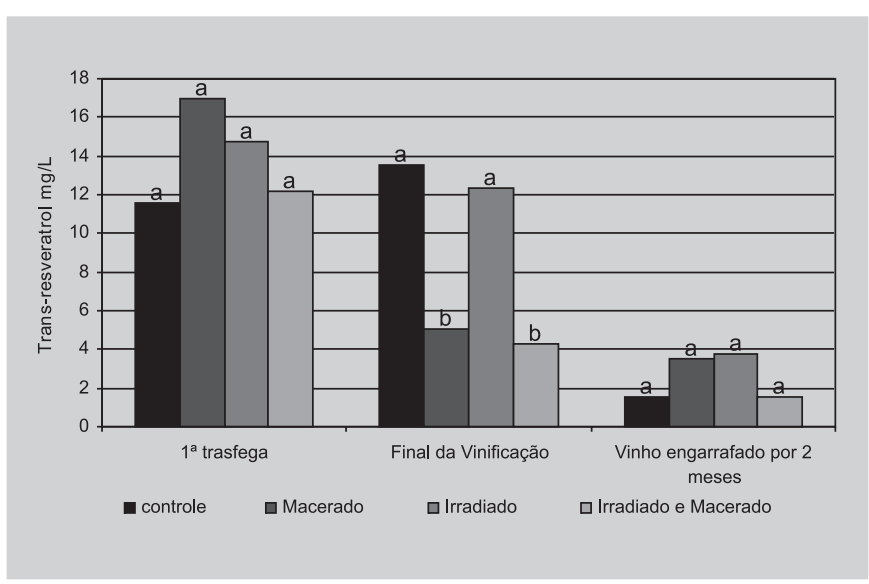

FIGURA 7 - Conteúdo de trans-resveratrol durante algumas etapas de vinificação do vinho de Cabernet Sauvignon (Safra 2003). Letras iguais não diferem significativamente a $5 \%$ de probabilidade pelo teste de Duncan.

A Figura 7 mostra, também, a etapa considerada como final da vinificação, ou seja, antes do engarrafamento do vinho, em que se observa diminuição de transresveratrol em quase todos os tratamentos, inclusive com diferença estatística entre eles. Os tratamentos com uvas não-irradiadas e sem maceração carbônica e de uvas somente irradiadas diferiu estatisticamente, dos tratamentos de uvas que sofreram somente maceração carbônica e daquele em que as uvas foram irradiadas e maceradas. Estas diminuições ocorrem devido, provavelmente, à precipitação dos sólidos solúveis, os quais carreiam polifenóis, entre eles, o resveratrol, ou pela polimerização dos estilbenos formando outros tipos de compostos (Sautter, 2003).

Os vinhos engarrafados e estocados por dois meses ( $3^{\text {a }}$ etapa do gráfico) tiveram as quantidades de transresveratrol diminuídas em relação às etapas anteriores, possivelmente pela precipitação dos bitartaratos de potássio e tartarato de cálcio, que, juntos, carrearam boas quantidade de trans-resveratrol. Permaneceram em média, 1,5 mg.L.-1 para vinhos produzidos com uvas irradiadas e com maceração carbônica e naqueles produzidos com uvas sem nenhum tipo de tratamento, apresentando resultado semelhante a Souto et al (2001), que encontrou 1,78 mg.L-1 em vinhos tintos brasileiros produzidos com uvas Cabernet Sauvignon. Segundo Jeandet et al. (1995), estas quantidades de trans-resveratrol devem permanecer constantes com o passar do tempo devido à estabilidade desta substância.

\section{CONCLUSÕES}

Os resultados deste trabalho indicam que, independente da técnica de vinificação utilizada, a evolução do conteúdo de trans-resveratrol foi ascendente, ocorrendo diferenças no 
final da fermentação. As amostras de vinhos com maceração carbônica apresentaram leve declínio devido à atmosfera de $\mathrm{CO}_{2}$ na qual ficaram armazenadas, inibindo, desta forma, a rota de formação do resveratrol. No final da fermentação principal, a concentração de trans-resveratrol foi de, aproximadamente, $15 \mathrm{mg} . \mathrm{L}^{-1} \mathrm{em}$ todos os tratamentos, chegando a 1,5 mg.L $\mathrm{L}^{-1}$, em média, ao fim do período de estocagem.

\section{ABSTRACT \\ Influence of the carbonic maceration on the levels of trans-resveratrol in cabernet sauvignon wine}

The moderate consumption of wine reduces the risks of heart diseases significantly. This effect is attributed to the polyphenols found in the wine, in special to resveratrol (3,5,4'-trihidroxistilbene) that it is a phytoalexin found in the various parts of the vine, including in the skin of the grape, as well as in other species of plants. Grapes of cultivar Cabernet Sauvignon had been submitted to the irradiation with ultra-violet light and carbonic maceration and after fermented. It was proceeded all collection from samples during the experiment, which later had been analyzed how much to the concentration of trans-resveratrol using the Liquid Chromatography of High Efficiency. The of the content of trans-resveratrol was ascendent during the vinification phases. Differences had occurred in the end of the fermentation where the samples of wines with carbonic maceration had presentes low decline, possibly for the $\mathrm{CO}_{2}$ atmosphere in which they were stored, in this way, inhibiting the formation of resveratrol. At the end of the main fermentation, the concentration of trans-resveratrol was $15 \mathrm{mg} . \mathrm{L}^{-1}$ in all treatments, arriving of $1.5 \mathrm{mg} \cdot \mathrm{L}^{-1}$, in average, at the end of storage period.

UNITERMS: Resveratrol. Irradiation U. Carbonic maceration. Cabernet Sauvignon.

\section{AGRADECIMENTOS}

Os autores agradecem à Vinícola Velho Amâncio de Santa Maria, RS, por ceder gentilmente as uvas Cabernet Sauvignon.

\section{REFERÊNCIAS BIBLIOGRÁFICAS}

ADRIAN, M.; JEANDET, A. C.; BREUIL, D.; LEVITE, S.; DEBORD, S.; BESSIS, R. Assay of Resveratrol and derivate stilbenes in wines by direct injection high performance liquid chromatography. Am. Enol. Vit., v. 51, n. 1, p. $37-41,2000$.
ARICHI, H.; KIMURA, Y.; OKUDA, H.; BABA, K.; KOZAWA, M.; ARICHI, S. Effects of stilbene components of the roots of Polygonum cuspidatum Sieb. Et zucc. on lipid metabolism. Chem. Pharm. Bull, v. 30, p. 1766-1770, 1982.

BRANDOLINI, V.; MAIETTI, A.;TEDESCHI, P.;DURINI, E.; VERTUANI, S.; MANFREDINI, S. Capillary electrophoresis determination, synthesis, and stability of resveratrol and related $3-O-b-\mathrm{D}$ glucopyranosides. J. Agri. Food Chem., v. 50, p. 74077411, 2002.

CANTOS, E.; ESPÍN, J. C.; FERNÁNDEZ, M. J.; OLIVA, J.; TOMÁS-BARBERÁN, F.A. Postharvest UV-C irradieted grapes as a potencial source for producing stilbene enriched red wines. J. Agri. Food Chem., v.51, p.1208-1214, 2003.

CANTOS, E.; GARCÍA-VIGUERA, C.; PASCUALTERESA, S. de; TOMÁS-BARBERÁN, F. A. Effect of postharvest ultraviolet irradiation on resveratrol and other phenolics of Cv. Napoleon table grapes. J. Agri. Food Chem., v. 48, p. 4606-4612, 2000.

DOR0ZYNSKI, A. Wine may prevent dementia. Br. Med.J., v. 314, p. 997, 1997.

GOLDBERG, D. M.; YAN, J.; NG, E.; DIAMANDIS, E. P.; KARUMANCHIRI,A.; SOLEAS, G.; WATERHOUSE, A. L. A global survey of Trans-resveratrol concentration in commercial wines. Am. J. Enol. Vit., v. 46, n. 2, p. 159 $165,1995$.

JEANDET, P.; BESSIS, R.; GAUTHERON, B. The production of resveratrol (3,5,4'-trihydroxystilbene) by grape berries in different developmental stages. Am. J. Enol. Vit., v. 42, p. 41-46, 1991.

JEANDET, P.; BESSIS, R.; MAUME, B. F.; MEUNIER, P.; PEYRON, D.; TROLLAT, P. Effect of enological practices on the resveratrol isomer content of wine. J. Agri. Food Chem., v. 43, p. 316-319, 1995.

KALLITHRAKA, S.; ARVANITOYANNIS, I.; ELZAJOULI, A.; KEFALAS, P. The application of an improved method for trans-resveratrol to determine the origin of Greek red wines. Food Chem., v. 75, p. 355-363, 2001. 
KIMURA, Y.; OHMINAMI,H.; OKUDA, H.; BABA, K.; KOZAWA, M.; ARICHI, S. Effects of stilbene components of roots of Polygonum spp. on liver injury in peroxidized oil fed rats. Planta Medica, v. 49, p. 51-54, 1983

LAMUELA-RAVENTOS, R. M.; ROMERO-PÉREZ, A. I. et al. Resveratrol and piceid levels in wine production and in finished wine. Wine nutritional and therapeutic benefits. Washington: American Chemical Society, 1997. p. 56-68. (ACS Simposium Series 661).

LAMUELA-RAVENTOS, R. M.; ROMERO PÉREZ, et al. Direct HPLC analysis of cis- and trans-resveratrol and piceid isomers in Spanish red Vitis vinifera wines. J. Agri. Food Chem., v. 43, n. 2, p. 281-283, 1995.

LANGCAKE, P.; PRYCE, R. J. The production of resveratrol and the viniferins by grapevines in response to ultraviolet irradiation. Phytochemistry, v. 16, p. 1193-1196, 1977.

MATTIVI, F.; NICOLINI, G. Influenza della technical de vinificazione sul contenudo di resveratrolo dei vini. $L$ ' Enotechnico, v. 29, p. 81-88, 1993.

OKUDA, T.; YOKOTSUKA, K. Trans-resveratrol concentrations in berry skins and wines from grapes grown in Japan. Am. J. Enol. Vit., v. 47, n. 1, p. 93-99, 1996.

RICARDO da SILVA, J. M.; CHEYNIER, V.; SAMSOM, A.; BOURZEIX, M. Effect of pomace contact, carbonic maceration, and hyperoxidation on the procyanidin composition of Grenache blanc wines. Am. J. Enol. Vit., v. 44, n. 2, p. 168-172, 1993.

RIZZON, L. A.; MIELE, A.; MENEGUZZO, J.; ZANUZ, . C. Efeito de três processos de vinificação sobre a composição química e a qualidade do vinho Cabernet Franc. Pesqui. Agropecu. Bras., Brasília, v. 34, n. 7, p. 1285-1293, 1999.
RODRÍGUEZ-DELGADO, M. A.; GONZÁLES, G.; PÉREZ-TRUJILLO, J. P.; GARCÍA-MONTELONGO, F. J. Trans-resveratrol in wines from the Canary Isands (Spain). Analysis by high performance liquid chromatography. Food Chem., v.76, p.371-375, 2002.

ROGGERO, J. P.; GARCIA PARRILLA, C. Effects of ultraviolet irradiation on resveratrol and changes in resveratrol and various of its derivates in the skins of ripening grapes. Sci. Alim., v. 15, p. 411-422, 1995.

SAUTTER, C. K. Avaliação da presença de resveratrol em suco de uva. Santa Maria, 2003. 130 p. [Dissertação de Mestrado. Universidade Federal de Santa Maria].

SAUTTER, C. K.; DENARDIN, S.; ALVES, A. O.; MALLMANN, C. A.; PENNA, N. G.; HECKTHEUER, L. H. R. Determinação de resveratrol em sucos de uva no Brasil. Cien. Tecnol. Al., v. 25, n. 3, p. 437-442, 2005.

SOLEAS, G. J.; GOLDBERG, D. M.; DIAMANDIS, E. P.; KARUMANCHIRI, A.; YAN, J.; NG, E. A derivatized gas chromatographic-mass spectrometric method for the analysis of both isomers of resveratrol in juice and wine. Am. J. Enol. Vit., v. 46, n. 3, p. 346-352, 1995.

SOLEAS, G. J.; DIAMANDIS, E. P.; GOLDBERG, D. M.; Resveratrol: Amolecule whose time has come? And gone? Clini. Biochem., v. 30, p. 91-113, 1997.

SOUTO, A. A.; CARNEIRO, M. C.; SEFERIN, M.; SENNA, M. J. H.; CONZ, A.; GOBBI, K. Determination of trans-resveratrol concentrations in Brazilians red wines by HPLC. J. Food Comp. Anal., v. 14, p. 441-445, 2001.

THRELFALL, R. T.; MORRIS, J. R.; MAUROMOUSTAKOS A., Effect of variety, ultraviolet light exposure, and enological methods on the trans-resveratrol level of wine. Am. J.Enol. Vit., v. 50, n. 1, p. 57-64, 1999.

TOMERA, J. F. Current knowledge of the health benefits and disadvantages of wine consumption. Trend in Food Sci. Technol., v. 10, p. 129-138, 1999.

Recebido para publicação em 05 de agosto de 2004. Aceito para publicação em 16 de fevereiro de 2007. 